\title{
NOTE OF APPRECIATION / NOTE DE RECONNAISSANCE
}

The success of the journal is dependent upon our reviewers. The Editors gratefully acknowledge the commitment and goodwill of all the reviewers who assisted us in the publication of Volume 90. Thank you.
Le succès du journal dépend du travail acharné de nos réviseurs. Le personnel de la rédaction est reconnaissant de leur volonté et leur nombreuses contributions qui ont rendu possible la sortie du volume 90. Merci.
Adidharma, $\mathrm{H}$.

Adronov, A.

Allouche, A.-R.

Amer, A.

Amri, $\mathrm{H}$.

Anbhule, $\mathrm{P}$.

Anderson, E.

Aoiz, F.J.

Asfour, A.-F.

Azizian, S.

Babua, S.

Back, T.

Bain, A.

Baldelli, S.

Banerjee, M.

Barrales, $\mathrm{P}$.

Barriault, L.

Batey, R.

Bazgir, A.

Beauchemin, A.

Bera, P.P.

Beran, K.

Berg, D.

Bergman, J.

Biehl, E.

Bigioni, T.

Bodwell, G.

Bohne, C.

Borges, Daniel

Bozzelle, J.

Breen Carmichael, T.

Breit, B.

Briand, G.

Britten, J.

Brown, A.

Brown, $P$.

Brown, S.

Bryce, D.

Buhse, T.

Bull, J.

Cademartiri, L.

Campbell, R.

Chang, H.-C.

Chawla, $\mathrm{H}$.
Chen, A.

Chen, B.

Cheng, J.-P.

Cheung, A.S.C.

Chu, T.

Cimas, A.

Clive, D.L.J.

Cooks, R.G.

Crutchley, R.

Cunha, S.

Dai, S.

Dake, G.

Das, $P$.

Davidkova, M.

De Lasa, $\mathrm{H}$.

Deka, R.C.

Demeter, A.

Dias, J.

Ding, Y.-H.

Dong, $\mathrm{R}$.

Dutton, $\mathrm{P}$.

Eckert, C.

El-Nahas, A.

Ellis, B.

Farrell, J.

Fishtik, I.

Fonseca Gurra, C.

Fortes, A.

Foti, M.

Francl, M.

$\mathrm{Fu}, \mathrm{Z}$.

Fyles, T.

Galliford, C.

Gao, Q.Y.

Gauld, J.

Ge, M.

Geer, L.

Ghambarian, M.

Ghandi, K.

Ghiviriga, I.

Gianturco, F.

Gibbs-Davis, J.

Gillies, E.

Glossman-Mitnik, D.
Gonzalez, R.D.

Gorelsky, S.

Gorner, H.

Gossage, R.

Gottardo, C.

Gravel, M.

Gray, M.

Green, J.

Gross, J.H.

Guthrie, P.

Hall, D.

Han, K.

Hasan, M.N.

Hayden, M.

Hayes, C.

Hayes, $P$.

Hernandez-Cordoba, M.

Hopkinson, A.

Hudson, R.

Hultin, $P$.

Iwabuchi, Y.

Jaroniec, M.

Jeung, G.H.

Joule, J.

Ju, X.

Kahr, B.

Kamal, A.

Kamitori, S.

Keesee, R.G.

Keillor, J.

Kelland, M.

Kerman, K.

Kerr, M.

Kertesz, M.

Kerton, F.

Khodabakhsh, N.

Kidwai, M.

Kirby, C.

Koivisto, B.

Kolluru, S.

Kovalchuk, N.

Krische, M.

Krol, E.

Kumar, A.
Kyriacou, G.

Lai, C.H.

Lalevee, J.

Leadbeater, $\mathrm{N}$.

Lee, $\mathrm{H}$.

Lee, S.-L.

Legault, C.

Leigh, W.

Lessard, J.

Li, G.-P.

Li, Y.-W.

Li, Yuanjun

Lin, $\mathrm{H}$.

Lindsey, J.

Lissi, E.

Liu, G.J.

Liu, H.

Liu, Y.F.

Liu, Yufang

Liu, Z.

Long, W.

Lowary, T.

Lucy, C.

Lyubimova, O.

Ma, Shengming

Ma, Shuhua

Macartney, D.

Macdonald, C.

Magee, D.

Mahmoud, N.

Mahwinney, R.

Maly, K.

Mariani, A.

Marques, J.M.C.

McLaurin, J.

McNaughton, D.

Meanwell, N.

Mebel, A.M.

Mehrkhodavandi, P.

Meyer, M.

Mezey, P.

Michaelides, S.

Miguad, M.

Mishra, B. 


\begin{tabular}{|c|c|c|}
\hline Mohammadi, A. & Rivard, E. & Wang, Suning \\
\hline Mohan, R.S. & Roberts, C. & Wang, Y. \\
\hline Montaut, S. & Rudolph, W. & Wang, Z. \\
\hline Moores, A. & Russo, N. & Ward, D. \\
\hline Mosquera, R. & Ryabov, A. & Weaver, D. \\
\hline Motherwell, W.B. & Rychnovsky, S. & Westcott, S. \\
\hline Nau, W. & Salahub, D. & Whitehead, D. \\
\hline Nemykin, V. & Sayari, A. & Williams, V. \\
\hline Nenadis, N. & Schaper, F. & Wilson, P. \\
\hline Nguyen-Dang, T. & Schreckenbach, G. & Woo, T. \\
\hline Nguyen, M.T. & Schuster, D. & Workentin, M. \\
\hline Nicasio, M. & Schwan, A. & Wright, T. \\
\hline Nitz, M. & Semenikhin, O. & Wu, G. \\
\hline Noël, J. & Shakib, F.A. & Wu, Y. \\
\hline Noolvi, M. & Sheppard, T. & Wuts, P. \\
\hline Nyanhongo, G. & Sherman, J. & Yamabe, S. \\
\hline Oleschuk, R. & Shi, D. & Yamakawa, T. \\
\hline Omanovic, S. & Shi, G. & Yang, D. \\
\hline Ono, T. & Singer, R. & Yang, Guangfu \\
\hline Ooms, K. & Slenczka, A. & Yang, Guanyu \\
\hline Palacios, F. & Smith, M. & Yang, M. \\
\hline Park, J.K. & Smith, S. & Yao, X. \\
\hline Paul Pezacki , J. & Sondhi, S.M. & Yeung, $\mathrm{K}$. \\
\hline Payne, N. & Sowa, Jr., J.R. & Yu, H.-Z. \\
\hline Pearson, J. & Spataru, T. & Yuan, J. \\
\hline Pedras, M.S. & Spino, C. & Yue, X.-F. \\
\hline Pekcan, O. & Spychaj, T. & Zard, S. \\
\hline Pelton, A. & Staroverov, V. & Zgierski, M. \\
\hline Pelton, R. & Stevenson, T. & Zhang, C. \\
\hline Penner, G. & Stolte, S. & Zhang, J. \\
\hline Persaud, K. & Sun, P. & Zhang, T. \\
\hline Peslherbe, G. & Sun, $X$. & Zhao, G.-J. \\
\hline Petitjean, A. & Swaddle, T. & Zoellner, R.W. \\
\hline Phenix, C. & Szalewicz, K. & Zou, S. \\
\hline Pimienta, V. & Szarek, W. & \\
\hline Pirola, C. & Terskikh, V. & \\
\hline Poirier, M.D. & Thompson, M. & \\
\hline Politzer, P. & Tian, M. & \\
\hline Popelier, P. & Tian, W.Q. & \\
\hline Posner, G. & Tiana, G. & \\
\hline Power, B. & Tomasi, S. & \\
\hline Puddephatt, R. & Tomoko, Y. & \\
\hline Purcaro, G. & Trapp, O. & \\
\hline Rabai, G. & Trouillas, P. & \\
\hline Rathore, R. & Trudel, S. & \\
\hline Rauk, A. & Tu, C.-H. & \\
\hline Ray, A. & Umoren, S. & \\
\hline Reddy, P. & Valero, R. & \\
\hline Reddy, R. & Varela-Alvarez, A. & \\
\hline Ren, Y. & Veinot, J. & \\
\hline Reynolds, W. & Verboom, W. & \\
\hline Richter, M.M. & Wagner, B. & \\
\hline Rimarcik, J. & Wang, Shengjie & \\
\hline
\end{tabular}

